\section{P465 THE ACCURACY TRANSCUTANEOUS BILIRUBINOMETERY DURING AND AFTER PHOTOTHERAPY IN PRETERM INFANTS}

1,2Ali Raba*, Jan Miletin 2,1 ${ }^{2}$ Anne O'Sullivan. 'UCD School of Medicine and Medical Sciences, Dublin, Ireland, Dublin, Ireland; ${ }^{2}$ Coombe Women and Infants University Hospital, Dublin, Ireland

\subsection{6/archdischild-2019-epa.801}

Background and aims Transcutaneous bilirubinometery (TcB) is a quick and painless method to guide the management of neonatal jaundice. Few studies have conducted on the effectiveness of TcB in preterm infants under phototherapy. The aim of the present study to examine the accuracy of TcB measurements during and after phototherapy in preterm infants.

Methods A prospective cohort study performed in the Coombe Women and Infants University Hospital, Dublin, Ireland. Preterm infants $\left(23^{+0}\right.$ to $36^{+6}$ weeks of gestation) born between June 2017 and May 2018, were enrolled in the study if they developed significant jaundice requiring phototherapy. TcB was measured from exposed (TcBu) and covered $(\mathrm{TcBc})$ areas within an hour of obtaining total serum bilirubin (TsB) samples. Correlation between $\mathrm{TcB}(\mathrm{TcBu}$ and $\mathrm{TcBc}$ ) and TsB were examined during and after phototherapy.

Result This study consisted of 196 jaundiced preterm infants (mean birth weight $( \pm$ SD) $1605 \mathrm{~g}( \pm 638)$, mean gestational age ( \pm SD) $30.4( \pm 3.2)$ weeks of gestation). We obtained 327 simultaneous measurements during the phototherapy phase and 137 pairs of readings after the discontinuation of phototherapy. There were weak correlations between TsB and TcB during phototherapy $(\mathrm{r}: 0.33, \mathrm{P}<0.0001$ in covered, $\mathrm{r}$ : 0.39 , $\mathrm{P}<0.0001$ in uncovered areas). However, post-phototherapy measurements showed a strong correlation between TsB and TcB (r: 0.86, P <0.0001).

Conclusion Measurement of $\mathrm{TcB}$ using a bed-type device is a reliable method to estimate bilirubin level in preterm infants after discontinuation of phototherapy. It cannot, however, be used as a substitute for TsB measurement during phototherapy in preterm infants

\section{P466 SGA EXTREMELY AND LOW PREMATURE INFANTS: FEATURES OF THE NEONATAL PERIOD}

Anton Yuditskiy*, Tatyana Kovalenko, Irina Petrova. Izhevsk State Medical Academy, Izhevsk, Russian Federation

10.1136/archdischild-2019-epa.802

Newborns with SGA are at risk for postnatal adaptation disorders and increased neonatal morbidity. This problem is of particular relevance in extremely and low premature infants.

Aim To evaluate the features of the neonatal period in extremely and low premature infants with SGA.

Materials and methods 42 premature infants with a gestational age of 28.0 [26.0; 30.0] weeks were examined: 18 - with SGA (group 1), 24 - with anthropometric parameters corresponding to the gestation period (group 2). The research program included an assessment of the clinical and metabolic indicators of postnatal adaptation, neonatal morbidity, and anthropometric dynamics by a corrected age of 1 month. Statistical processing of the results: the frequency of symptoms $(\%)$, the median with the definition of the interquartile interval, the nonparametric criterion $\chi 2$.
Results The median mass and length at birth in children of group 1 corresponded to $650.0[610.0 ; 932.0] \mathrm{g}$ and 34.0 [31.0; 36.0] cm, 2 groups - 1090.0 [866.0; 1393.0] g (p $<0.01)$ and $36.0[33.0 ; 40.5] \mathrm{cm}(\mathrm{p}<0.05)$. The course of the neonatal period in extremely and low premature infants is characterized by postnatal adaptation disorders. In the case of SGA, the differences relate to the frequency of intrapartum asphyxia (group $1-88.9 \%$, group $2-58.3 \%$, p <0.05), acute adrenal insufficiency $(44.4 \%$ and $16.6 \%, \mathrm{p}<0,05)$, pathological hyperbilirubinemia $(38.9 \%$ and $8.3 \%, \mathrm{p}<0.05)$, edema $(33.3 \%$ and $8.3 \%, \mathrm{p}<0.05)$. All patients had pathology of the neonatal period. In SGA were more often recorded intraventricular hemorrhages with the formation of occlusive hydrocephalus (group $1-33.3 \%$, group $2-8.3 \%$, p <0.05), sepsis $(33.3 \%$ and $4.2 \%, p<0,05)$, congenital pneumonia $(72.2 \%$ and $33.3 \%, \mathrm{p}<0.05)$ and bronchopulmonary dysplasia $(50.0 \%$ and $16.7 \%, \mathrm{p}<0.05)$. However, in patients with SGA, the disease was more severe: intensive therapy was needed in 18 patients of group 1 and 24 in group 2. The duration of intensive therapy in preterm patients with SGA was significantly higher than in group $2: 28.0[14.5 ; 37.5]$ and $8.0[6.0 ; 10.7]$ days $(\mathrm{p}<0.01)$. By the corrected age of 1 month, the indicators of mass and length of the body in the very premature with SGA remained unsatisfactory: 3,380.0 $[2,773.0 ; 3627.0] \mathrm{g}(-2.3 \mathrm{SD})$ and $50.0[44.0 ; 52.5] \mathrm{cm}(-1.9$ $\mathrm{SD})$.

Conclusion Prematurity and SGA have an adverse potentiating effect on the course of the neonatal period.

\section{P467 NUTRITION AUDIT- ARE WE LOSING THE PLOT?}

Katarina Stefkova*, David Cummins, Alison Walker. Royal Jubilee Maternity Hospital, Belfast, UK

\subsection{6/archdischild-2019-epa.803}

Background and aims Growth measurement and documentation is an essential part of practice in neonatology, as regular measuring of growth parameters leads to early recognition of possible poor growth. This means action can be taken promptly, reducing long term morbidity and mortality.

The Royal College of Paediatrics and Child Health have produced guidelines on growth measurement, with NICU in RJMH having additional guidelines specific to the unit.

Our aim was to audit the compliance with the guidelines in $\mathrm{RJMH}$, and to find ways of improving practice.

Methods We have audited growth charts on BadgerNet of 58 babies, which was the total number of inpatient babies during the month of November. This was done as a retrospective audit.

Results $100 \%$ of babies audited had birth weight plotted on a correct chart with EDD marked on the chart. We attributed this to the fact that RJMH uses BadgerNet, which is an electronic system that automatically generates a growth chart and plots the birth weight. Birth head circumference was plotted on $69 \%$ of the babies.

Out of the babies that resided in the unit over 2 weeks, $90 \%$ had a second weight noted by 2 weeks, and $72 \%$ had repeat head circumference noted within 2 weeks. Only $28 \%$ of babies had their weight plotted twice weekly thereafter, and $22 \%$ of babies had weekly head circumference plotted.

Conclusions Our conclusion from the audit is that initial growth parameters are performed well, however ongoing 
growth measurement and growth chart plotting requires improvement.

\section{P468 ADMISSION TEMPERATURE OF OUTBORN NEWLY BORN INFANTS FOLLOWING NEONATAL TRANSPORT: A LITERATURE REVIEW}

${ }^{1,2}$ Katie Cunningham*, PF Colm ${ }^{1,2} 0^{\prime}$ Donnell, 1,2 Lisa K McCarthy. 'National Maternity Hospital, Holles Street, Dublin, Ireland; ${ }^{2}$ School of Medicine, University College Dublin, Dublin, Ireland

\subsection{6/archdischild-2019-epa.804}

Background Infants born at hospitals without neonatal intensive care (NICU) facilities that are transferred to a NICU after birth are referred to as outborn. Ideally all outborn infants are transferred by dedicated neonatal transport services. Abnormal temperature in newborn infants is associated with increased morbidity and mortality. It is an important function of transport teams to maintain normal body temperature $\left(36.5^{\circ} \mathrm{C}-37.5^{\circ} \mathrm{C}\right)$ during transport. The aim of this review was to assess the prevalence of abnormal admission temperature in outborn infants following neonatal transport.

Methods We searched PubMed (NCBI) and Web of Science from inception to April 2018 using the following search terms; (newborn OR neonate OR 'new born' OR 'premature infant' OR 'preterm infant') AND ((temperatrure OR hypothermia) NOT ('therapeutic hypothermia' OR 'passive hypothermia' OR 'hypothermia, induced')) AND ('interhospital transport' OR 'inter-hospital transport' OR 'interfacility transport' OR 'inter-facility transport' OR 'neonatal transport'). We planned to include randomised trials, case-control studies, prospective and retrospective cohort studies and case series. We excluded studies of infants undergoing therapeutic hypothermia and articles published in a language other than English.

Results The initial search identified 66 articles. Twenty articles met inclusion criteria. A further 9 were identified from reference lists. Studies spanned a 45-year period (1973-2018). Definitions of hypo- and hyper-thermia varied considerably, as did patient inclusion criteria with combinations of preterm and term infants, and infants with or without congenital anomalies. Specialised transport services featured in 16 (55\%) studies, with a reported incidence of hypothermia ranging from $0.2 \%-70 \%$. There was significant correlation between birth weight and admission temperature, and gestation and admission temperature, such that incidence of hypothermia on admission increased with decreasing weight and gestation.

The reported incidence of hyperthermia ranged from 0\%$19 \%$. Death was more commonly seen in infants who were hypothermic on admission, with moderate hypothermia $\left(32^{\circ} \mathrm{C}\right.$ $36^{\circ} \mathrm{C}$ ) identified as an independent risk factor for death. Mortality rates appeared to improve when transfers were performed by neonatal transport teams.

Conclusion Maintaining normal body temperature in outborn newly born infants during neonatal transport continues to pose challenges for neonatal transport teams. Risk factors for hypothermia and associated adverse outcomes include prematurity, very-low birth weight and transfer by non-specialised transport teams. This is especially so in developing countries without established transport services. Hyperthermia also occurs, but is less frequently reported. Using standardised definitions, further study examining temperature in neonatal transport is required.

\section{P469 \\ OUTCOMES OF BABIES WITH BIRTH WEIGHT UNDER 500 GRAMS IN A NEONATAL INTENSIVE CARE UNIT}

Fateh Singh, Nikita Gulati, Niranjana Mahalingam, Shree Vishna Rasiah*, Anju Singh. Birmingham Women's and Children's NHS foundation Trust, Birmingham, UK

\subsection{6/archdischild-2019-epa.805}

Background Determining the limits of viability for resuscitating a baby is important to avoid un-necessary interventions. Establishing these boundaries is an ethical dilemma that has been under constant debate. There is greater clarity and guidance on gestational age for limits of viability as compared to weight criteria.

Aims To evaluate the in-hospital mortality and morbidity in babies born under 500 grams.

Methods Retrospective data was collected from Badger database over a 9 year period from April 2009 to March 2018 looking at the mortality and morbidity in babies with birth weight under 500 grams in a neonatal intensive care unit.

Results There were 28 babies in the study cohort. The mean gestational age was 25 weeks (range 22+6-28+4) with 46\% survival. There were 12 males and 16 females, with higher mortality in males ( $75 \%$ versus $38 \%$ in females). 3 babies had oesophageal perforation with nasogastric tube which was managed conservatively. In the survivors 8 babies had necrotising enterocolitis and none required surgery. There were 2 surviving babies with grade 2 or above intraventricular haemorrhage and 2 babies required laser therapy for retinopathy of prematurity. 2 of the surviving babies (15\%) were discharged home on oxygen. The average hospital stay for the surviving infants was 111 days. Of the babies who died, 4 had necrotising enterocolitis and 8 babies with grade 2 or above intraventricular haemorrhage.

Conclusion Despite advances in neonatal practice, our single center data shows that the outcome of babies born with a birth weight of under 500 grams remains very poor. The mortality was significantly higher in the male babies. The management of these babies is challenging with multiple comorbidities requiring significant neonatal resources.

\section{P470 SPONTANEOUS INTESTINAL PERFORATION IN AN EXTREMELY LOW BIRTH WEIGHT INFANT: A CASE REPORT}

${ }^{1}$ Ayşe Pervanlar, ${ }^{2}$ Ayça Sözen, ${ }^{1}$ Tuğba Erener Ercan*, 'David Terence Thomas. ${ }^{1}$ Maltepe University Faculty of Medicine, Istanbul, Turkey; ${ }^{2}$ Akdağmadeni State Hospital, Yozgat, Turkey

10.1136/archdischild-2019-epa.806

Spontaneous intestinal perforation(SIP) usually occurs in the first 10 days of life primarily in preterm infants with very low birth weight (VLBW) and extremely low birth weight (ELBW). The risk is $2-3 \%$ and $5 \%$ in VLBW infants and ELBW infants, respectively.

Herein, we present a case of a $410 \mathrm{~g}$ female infant born at 25 weeks of gestational age who developed SIP on the 7 th postnatal day. The patient was born by emergent cesarean delivery after the diagnosis of chorioamnionitis in the mother. She was intubated in the delivery room due to insufficient respiratory effort and surfactant was administered. The infant was admitted to the neonatal intensive care unit (NICU) and she was mechanically ventilated in SIMV mode. Her 